www.nature.com/clinicalpractice/neuro

Abelson and colleagues investigated SLITRK1 on chromosome 13 because of its proximity to a DE NOVO CHROMOSOME INVERSION identified in a child diagnosed with TS, who had no family history of TS or related conditions. The gene was known to be expressed at a relatively high level in brain areas associated with TS. The researchers carried out intensive screening for mutations in SLITRK1 in 174 individuals with TS, and identified one individual who had a frameshift mutation in the coding region - a mutation that did not appear in 3,600 control chromosomes. Screening of SLITRK1 in 253 controls also revealed no truncating or deleterious mutations in the coding region.

The researchers identified a noncoding sequence variant (var321) that was present in two individuals with TS, but absent from 4,296 control chromosomes. This mutation lies within a predicted binding site on the SLITRK1 messenger RNA for a microRNA called hsa-miR-189. In vitro studies indicate that this microRNA can repress SLITRK1 protein synthesis, and that alteration in the interaction with SLITRK1 messenger RNA caused by the identified mutations is likely to be contributing to TS in these patients.

The authors conclude that further study of SLITRK1 might aid our understanding of the cellular and molecular basis for TS, and help to identify the pathway underlying the disease pathogenesis.

Christine Kyme

Original article Abelson JF et al. (2005) Sequence variants in SLITRK1 are associated with Tourette's syndrome. Science 310:317-320

\section{Novel approach to immunotherapy for Alzheimer's disease}

A novel potential vaccination treatment for Alzheimer's disease (AD) was recently described by Frenkel and colleagues. This new approach avoids the use of anti-amyloid- $\beta$ (anti-A $\beta$ ) antibodies, which were found to cause meningoencephalitis in clinical trials.

$A \beta$-peptide fibrils are thought to have a key role in $A D$ pathogenesis, and the researchers carried out a series of experiments in $A D$ mouse models. They investigated the potential of glatiramer acetate (GA; currently used to treat multiple sclerosis), administered nasally in combination with a proteosome-based mucosal adjuvant known to be well tolerated in humans, to clear $(A \beta)$ fibrils. Immunization with GA and the proteosome-based adjuvant resulted in a $73 \%$ reduction in total brain $A \beta$ levels compared with controls $(P<0.001)$. The combination of GA and the mucosal adjuvant had the advantage of producing potent therapeutic effects via a treatment that could be administered nasally rather than requiring invasive parenteral administration.

Immunohistochemical analysis revealed that animals treated with the nasal vaccination developed activated microglia that colocalized with $A \beta$ plaques, and there was a strong correlation between the amount of microglial activation and the observed reduction in $A \beta$ fibrils.

The authors propose that their results suggest a novel approach to immune therapy for AD that uses compounds that have been safely tested or used in humans. They note, however, that GA and the adjuvant have not yet been tested together in humans.

Christine Kyme

Original article Frenkel D et al. (2005) Nasal vaccination with a proteosome-based adjuvant and glatiramer acetate clears $\beta$-amyloid in a mouse model of Alzheimer disease. J Clin Invest 115: 2423-2433

\section{Statin therapy correlates with improved outcomes following carotid endarterectomy}

Removal of atherosclerotic plaques by carotid endarterectomy can prevent stroke in symptomatic patients. In asymptomatic patients, however, the risk of complications during surgery might outweigh the benefit of this treatment. Statins are lipid-lowering drugs that also prevent stroke and are associated with fewer adverse outcomes in noncardiac surgery.

In an observational study, Kennedy et al. tested whether statin use at the time of hospital admission was associated with improved outcomes following carotid endarterectomy in symptomatic and asymptomatic subjects. Out of 3,360 patients undergoing carotid endarterectomy, 3,283 were considered suitable for analysis. Statistical analysis showed that, in symptomatic patients, statin use was associated with decreased rates of
GLOSSARY

DE NOVO CHROMOSOME INVERSION

A chromosome inversion that has not been inherited 